\title{
DIFFERENTIAL EXPRESSION OF RADIATION RESPONSE GENES IN SPLEEN, LUNG, AND LIVER OF RATS FOLLOWING ACUTE OR CHRONIC RADIATION EXPOSURE
}

\author{
${\text { Hee } \mathrm{Jin}^{*} \text {, Yeung Bae Jin }}^{\dagger}{ }^{\ddagger}$, Ju-Woon Lee ${ }^{\dagger}$, Jae-Kyung Kim ${ }^{\dagger}$, and Yun-Sil Lee* \\ *Graduate School of Pharmaceutical Sciences, Ewha Womans University, Seoul Korea, \\ ${ }^{+}$Advanced Radiation Technology Institute, Korea Atomic Energy Research Institute, Jeongeup, Korea, \\ ${ }^{\ddagger}$ These authors contributed equally to the study.
}

Received November 28, 2014 / 1st Revised December 23, 2014 / Accepted for Publication December 28, 2014

We analyzed the differential effects of histopathology, apoptosis and expression of radiation response genes after chronic low dose rate (LDR) and acute high dose rate (HDR) radiation exposure in spleen, lung and liver of rats. Female 6-week-old Sprague-Dawley rats were used. For chronic low-dose whole body irradiation, rats were maintained for 14 days in a ${ }^{60} \mathrm{Co}$ gamma ray irradiated room and received a cumulative dose of $2 \mathrm{~Gy}$ or $5 \mathrm{~Gy}$. Rats in the acute whole body exposure group were exposed to an equal dose of radiation delivered as a single pulse $\left({ }^{137} \mathrm{Cs}\right.$-gamma). At 24 hours after exposure, spleen, lung and liver tissues were extracted for histopathologic examination, western blotting and RT-PCR analysis.

1. The spleen showed the most dramatic differential response to acute and chronic exposure, with the induction of substantial tissue damage by HDR but not by LDR radiation. Effects of LDR radiation on the lung were only apparent at the higher dose (5 Gy), but not at lower dose (2 Gy). In the liver, HDR and LDR exposure induced a similar damage response at both doses. RT-PCR analysis identified cyclin $G 1$ as a LDR-responsive gene in the spleen of rats exposed to $2 \mathrm{~Gy}$ and $5 \mathrm{~Gy}$ gamma radiation and in the lung of animals irradiated with 5 Gy.

2. The effects of LDR radiation differed among lung, liver, and spleen tissues. The spleen showed the greatest differential effect between HDR and LDR. The response to LDR radiation may involve expression of cyclin G1.

Keywords: Radiation response genes, Acute exposure, Chronic exposure, Differential expression

\section{INTRODUCTION}

High doses of ionizing radiation (IR) are often harmful to living organisms. However, at a low dose or low dose rate (LDR), radiation is not as harmful as once thought. Chronic LDR exposure to IR may have different effects than an equal dose of acute radiation. Reported effects of LDR radiation include stimulation of growth rate [1], enhancement of survival after lethal high-dose irradiation $[2,3]$, prolongation of life span,

Corresponding author : Yun-Sil Lee, yslee0425@ewha.ac.kr

College of Pharmacy, Graduate School of Pharmaceutical Sciences, Ewha

Womans University, Seoul 120-750, Korea
$[4,5]$ activation of immune functions [6-9], increased resistance to oxygen toxicity [10], improvement in mouse behavior [11], and disease prevention or cure [12-14]. Therefore, dose rate is a very important factor determining the biological consequences of IR.

Many natural and man-made sources of LDR-IR affect humans through occupational, medical, and environmental exposures. The carcinogenic effects of LDR exposure in humans have been reviewed [15]. However, a LDR strategy of IR is commonly employed as an effective radiotherapeutic strategy (termed brachytherapy) for some cancers including prostate, gynecologic, lung, breast, head and neck, 
anal/rectal, and esophageal cancers, as well as soft tissue sarcomas. Indeed, the use of permanent LDR brachytherapy is a common treatment approach to low risk, early stage prostate cancer, in which radioactivity of a permanently implanted iodine or palladium seed decays over several months within the gland. LDR brachytherapy is also sometimes used in the treatment of coronary artery disease to prevent restenosis after angioplasty. Hence, a better understanding of the cellular response to LDR-IR is of clinical interest.

Cell and tissue sensitivity to IR is related to expression patterns of many endogenous genes. Various stimuli, including IR, produce genetic damage and changes in gene expression. Important factors determining outcome include whether the cell dies, the accuracy of repair of the radiation-induced damage, or whether the cell response leads to cell transformation and cancer. Whether the damage leads to cell death or survival is critical because death of a damaged cell removes a potential problem. If the cell does not die, it may acquire genomic instability and give rise to a population of cells with abnormally high susceptibility to gene and chromosomal instability, mutations, and other delayed effects [16-18]. Despite many epidemiologic studies addressing the effects of LDR radiation in exposed humans and experimental investigations on model animals and cells, the fundamental effects of radiation exposure remain unclear.

Previous studies have identified genes that are overexpressed in human peripheral blood lymphocytes [19] and radiation-inducible genes and proteins in several organs $[20,21]$. We previously investigated the potential use of these genes as candidate blood biomarkers for radiation exposure, particularly local exposure [21].

This study explored whether total body exposure to chronic LDR or acute HDR irradiation induces differential expression of radiation-responsive genes in spleen, lung, and liver tissues of rats. We compared histopathologic changes, induction of apoptosis, and gene expression in these tissues after acute or chronic irradiation at total doses of 2 Gy and 5 Gy.

\section{MATERIALS AND METHODS}

\author{
Animals \\ Female 6-week-old Sprague-Dawley (SD) rats were \\ purchased from ORIENT-BIO (Seoul, Korea) and \\ were housed in specific pathogen-free barrier
}

facilities. The temperature and relative humidity in the exposure facility was maintained at $22 \pm 2{ }^{\circ} \mathrm{C}$ and $50 \pm 10 \%$, respectively, and both conditions were continuously monitored. Fluorescent lighting was provided for 12 hours each day. The studies were performed according to guidelines for use and care of laboratory animals and were approved by the Institutional Animal Care and Use Committee (IACUC) of the Korea Atomic Energy Research Institute (KAERI; authorization number KAERI- IACUC-2012-022).

\section{Experimental design and irradiation}

The experimental design is shown in Fig. 1. Twenty five rats were randomly divided into five groups. Two groups were irradiated with the high dose rate, two groups were irradiated with the low dose rate, and one group was the non-irradiated control. Whole body HDR radiation was generated using a ${ }^{137} \mathrm{Cs}$ source $\left(1 \mathrm{~Gy} \cdot \mathrm{min}^{-1}\right.$, MDS Nordion International, Ottawa, ON, Canada). Whole body LDR radiation was generated using a gamma phytotron $\left({ }^{60} \mathrm{Co}\right.$ source, $150 \mathrm{TBq}$ of capacity; Atomic Energy of Canada, Ottawa, ON, Canada). Both doses were generated at the Korea Atomic Energy Research Institute (KAERI). LDR irradiation exposure was sustained for 14 days at dose rates of 5.95 and 14.88 $\mathrm{mGy} \cdot \mathrm{h}^{-1}$ (total dose 2 Gy and $5 \mathrm{~Gy}$, respectively). Twenty- four hours after completion of radiation treatment the rats were sacrificed and tissue samples were collected.

\section{Tissue preparation}

Twenty-four hours after irradiation the animals were killed and organs were promptly harvested. A portion of each tissue was fixed in $10 \%$ neutral buffered formalin and embedded in paraffin. Sections 3-5 $\mu \mathrm{m}$ in thickness were stained with hematoxylin and eosin (H\&E). The remainder of the tissue was preserved in liquid nitrogen for later RNA preparation.

\section{Western blot analysis}

Organ tissues were homogenized in PRO-PREP ${ }^{\mathrm{TM}}$ lysis buffer (iNtRON, Gyeonggi-Do, Korea). Protein concentration was determined by the Bradford method (Bio-Rad, Hercules, CA, USA). The protein in denatured samples was resolved by $10-15 \%$ sodium dodecyl sulfate-polyacrylamide gel electrophoresis (SDS-PAGE) and electrotransferred to nitrocellulose membranes. The membranes were immunoblotted with the appropriate primary antibody and treated with horseradish peroxidase-conjugated anti-IgG secondary antibody (Santa Cruz Biotechnology, TX, 
Table 1. Gene List Used in RT-PCR Analysis.

\begin{tabular}{|c|c|c|c|}
\hline & Gene name & Function & Primer sequence \\
\hline \multirow{2}{*}{1} & \multirow{2}{*}{ protein tyrosine kinase } & \multirow{2}{*}{ Tyrosine phosphorylation } & Sense: ATTCAGCGCGTTACCATTTC \\
\hline & & & Antisense: TGGCCCACCTATGGAAAATA \\
\hline \multirow{2}{*}{2} & \multirow{2}{*}{ sialyltransferase } & \multirow{2}{*}{ Glycosyltransferase } & Sense: GCGCTTCCTCAAGGACAGTTTGTA \\
\hline & & & Antisense: CAGAAAGGGTGGCTTTCCCAAACA \\
\hline \multirow{2}{*}{3} & \multirow{2}{*}{$\mathrm{Cu} / \mathrm{Zn}$ superoxide dismutase } & \multirow{2}{*}{ Antioxidant enzyme } & Sense: CGGTGAACCAGTTGTGTTGT \\
\hline & & & Antisense: CACCTTTGCCCAAGTCATCT \\
\hline \multirow{2}{*}{4} & \multirow{2}{*}{ phosphoglycerate kinase 1} & \multirow{2}{*}{ Phosphoryltransferase } & Sense: GAATGCAAAGACTGGCCAAGCTAC \\
\hline & & & Antisense: CCAGTGCTCACATGGCTGACTTTA \\
\hline \multirow{2}{*}{5} & \multirow{2}{*}{ transaldolase 1} & \multirow{2}{*}{$\begin{array}{l}\text { Transfer of a three-carbon ketol } \\
\text { unit }\end{array}$} & Sense: TCCACTGCAACATGACACTGCT \\
\hline & & & Antisense: TCCGCTCCAACTTTATGGCATCAG \\
\hline \multirow{2}{*}{6} & \multirow{2}{*}{ cyclin G1 } & \multirow{2}{*}{ Cell cycle regulation } & Sense: GGTCTGTGGCCTGAAACTGATTGA \\
\hline & & & Antisense: CAAATGGCAAGGTCTCCCGAATGA \\
\hline \multirow{2}{*}{7} & \multirow{2}{*}{ hsp25 } & \multirow{2}{*}{ Chaperone } & Sense: ATGAGTGGTCTCAGTGGTTCAGC \\
\hline & & & Antisense: CTCGAAAGTGACCGGAATGGTGAT \\
\hline \multirow{2}{*}{8} & \multirow{2}{*}{ hsp70 } & \multirow{2}{*}{ Chaperone } & Sense: TGCTGACCAAGATGAAGGAGATCG \\
\hline & & & Antisense: GCTTGTTCTGGCTGATGTCCTTCT \\
\hline \multirow{2}{*}{9} & \multirow{2}{*}{ hsp90 } & \multirow{2}{*}{ Chaperone } & Sense: ACAAGCAAGACCGAACCCTCACTA \\
\hline & & & Antisense: TGTTCCACGACCCATTGGTTCA \\
\hline \multirow{2}{*}{10} & \multirow{2}{*}{ hsf1 } & \multirow{2}{*}{ Transcriptional factor } & Sense: TGTGCGGCAGCTCAACATGTAT \\
\hline & & & Antisense: GACTGCACCAGTGAGATCAGGAAT \\
\hline
\end{tabular}

USA). The primary antibodies used were: $\beta$-actin (Santa Cruz Biotechnology, TX, USA) for loading control and antibody against cleaved caspase 3 (Cell Signaling Technology, MA, USA) for a measure of apoptosis. Immunoblotted proteins were visualized using enhanced chemiluminescence (EzWestLumi, Taito-ku, Tokyo, Japan).

\section{Total RNA isolation}

Total RNA was extracted from tissue using TRIreagent (Molecular Research Center, Cincinnati, $\mathrm{OH}$, USA). Each $0.1 \mathrm{~g}$ sample was homogenized in $1 \mathrm{~mL}$ of TRIreagent for $10 \mathrm{~min}$. The homogenized sample was centrifuged and the RNA was extracted from the supernatant according to the manufacturer's protocol. RNA status was always checked and degraded ones were excluded for performing RT-PCR.

\section{$R T-P C R$}

RNA (1-5 $\mu \mathrm{g})$ was reverse transcribed into cDNA using a ReverTra Ace ${ }^{\circledR}$ qPCR RT Kit (TOYOBO, Kita-ku, Osaka, Japan) following the manufacturer's protocol. A total of $1 \mu \mathrm{L}$ of the cDNA mixture was used for enzymatic amplification. PCR was performed with $1.75 \mathrm{mM} \mathrm{MgCl}_{2}, 0.2 \mathrm{mM}$ dNTPs, Taq polymerase (GenDEPOT, Barker, TX, USA), and 0.5 $\mu \mathrm{M}$ of each primer for target genes in a thermal cycler (Bio-Rad, Hercules, CA, USA). The cycling conditions were denaturation at $94^{\circ} \mathrm{C}$ for $5 \mathrm{~min}$, followed by $25-30$ cycles of denaturation at $94^{\circ} \mathrm{C}$ for $20 \mathrm{~s}$, annealing at $58^{\circ} \mathrm{C}$ for $10 \mathrm{~s}$, and extension at $72^{\circ} \mathrm{C}$ for 30 s. Primers are shown in Table 1. PCR products were separated by $2 \%$ agarose gel electrophoresis and stained with SafePinky DNA Gel Staining Solution (GenDEPOT, Barker, TX, USA). The products were quantified using an image analyzer with the Micro Computer Imaging Device (MICD) software program (Image Research, St. Catharines, ON, Canada).

\section{Statistical analyses}

Data are expressed as mean \pm standard deviation (SD). Statistical significance was determined using Student's $t$-test.

\section{RESULTS}

\subsection{Acute HDR radiation induces more serious organ damage than chronic LDR radiation, particularly in the spleen}

To compare the radiation damage after HDR IR or LDR IR we selected the spleen, lung, and liver because they are particularly radiation-sensitive organs $[20,22,23]$. Spleen tissue of rats exposed to LDR of 2 Gy was similar to that of control rats except for some scattered necrosis. HDR using a dose of 2 Gy induced serious necrosis and mild hemorrhage in the red pulp. LDR with a dose of 5 Gy induced an irreg- 


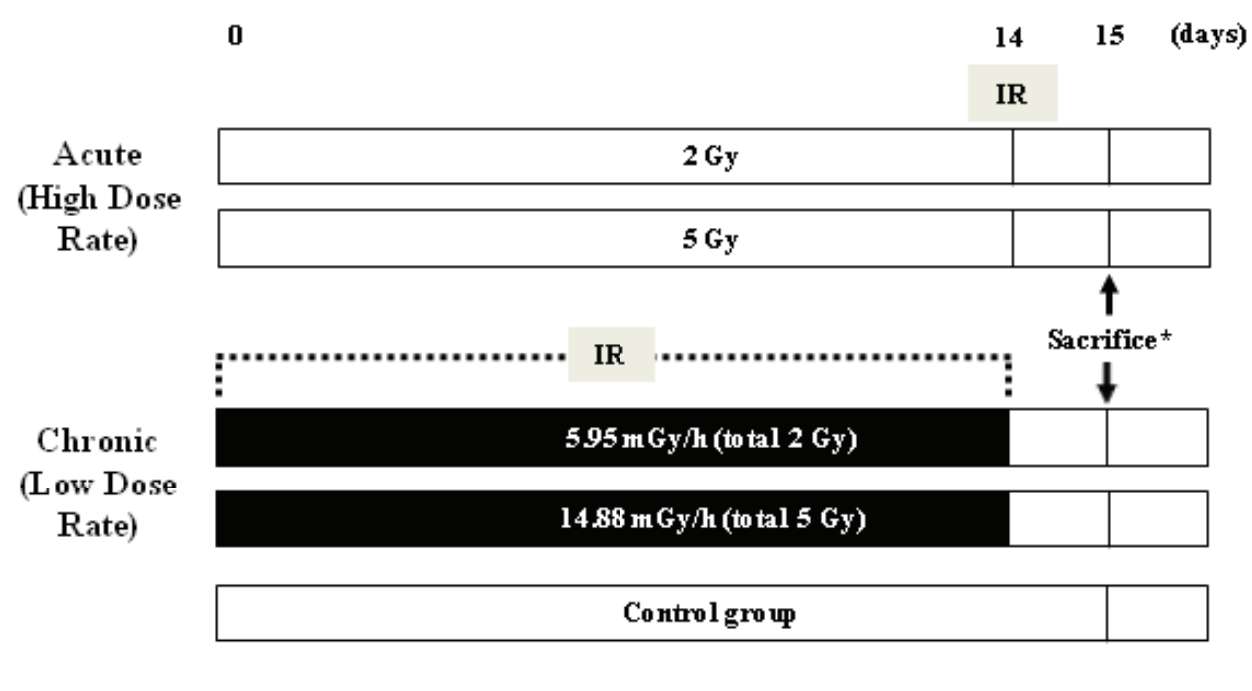

* Sacrifice: 24 hr after IR

Fig. 1. Schematic of the experimental procedure.

ular pattern involving both white and red pulp and the periarteriolar lymphoid sheaths, together with some necrosis, whereas HDR using a dose of 5 Gy produced massive splenic hemorrhage and highly expanded red pulp accompanied by destroyed borderline white pulp. LDR exposure using 2 Gy did not induce any histological changes in lung tissue compared with control lung tissue. Lungs of animals exposed to 5 Gy LDR, 2 Gy HDR, and 5 Gy HDR showed thickening of the alveolar walls by lymphocytes and macrophages and hyperplasia of lymphatic follicles in the respiratory tract. HDR with 5 Gy induced massive hemorrhage and severe infiltration of inflammatory cells in lung tissue. Liver tissue showed no pathologic difference between HDR or LDR radiation at a dose of $2 \mathrm{~Gy}$ and the control. Necrotic hepatocytes and cell swelling were seen in liver tissue following exposure of rats to both LDR and HDR at a dose of 5 Gy. Some of the cells were enucleated and displayed expanded sinusoids. Therefore LDR and HDR radiation produced similar effects in liver tissue (Fig. 2). These data indicate that LDR and HDR induced different degrees of damage in different organs, even at the same total dose, with the spleen showing the greatest difference between HDR and LDR.

\subsection{Cleavage of caspase 3 by HDR or LDR radiation}

To determine whether pathologic differences between HDR and LDR exposure correlated with the induction of apoptosis, we examined caspase 3 activation by western blot analysis of caspase 3 cleavage in LDR- or HDR-irradiated liver, lung, and spleen tissues. In spleen tissue, HDR for 2 Gy and 5 Gy induced increased cleavage of caspase 3 , whereas LDR at both doses induced minimal caspase 3 cleavage. In the lung, there was no difference in the cleavage of caspase 3 between unirradiated control and LDR exposure at doses of 2 Gy and 5 Gy. Similarly, 2 Gy HDR did not induce caspase-3 cleavage but 5 Gy HDR induced significant cleavage of caspase 3 . Therefore, at a dose of 5 Gy there was a significant difference between LDR and HDR exposure in lung tissue. In the liver, both HDR and LDR exposure at doses of 2 Gy and 5 Gy induced cleavage of caspase 3. Liver tissue did not show appreciable differences between LDR and HDR exposure at the same dose. Therefore caspase 3 activation was differentially induced by HDR and LDR exposure at both 2 Gy and 5 Gy in the spleen and at 5 Gy only in the lung (Fig. 3).

\subsection{Differential gene expression patterns in the spleen according to LDR or HDR exposure}

To determine whether the differences in histopathology and the induction of caspase 3 cleavage between the LDR and HDR regimens was mediated by altered gene expression, we examined several previously identified radiation-responsive genes: $p t k$, st6gall, sod, hsp25, hsp70, hsf1, ta1, and cyclin G1 [19-21, 24]. In spleen tissue, which showed the most significant difference in histopathology and caspase 3 activation between LDR and HDR exposure, expression of $h s p 25$ and cyclin G1 was significantly increased by LDR exposure at a dose of 2 Gy and expression of $h s p 90$ and tal was significantly increased by HDR at a dose of 2 Gy. Comparison of LDR and HDR exposure at 5 Gy revealed that LDR increased the expression of sod and cylcin G1 and decreased 


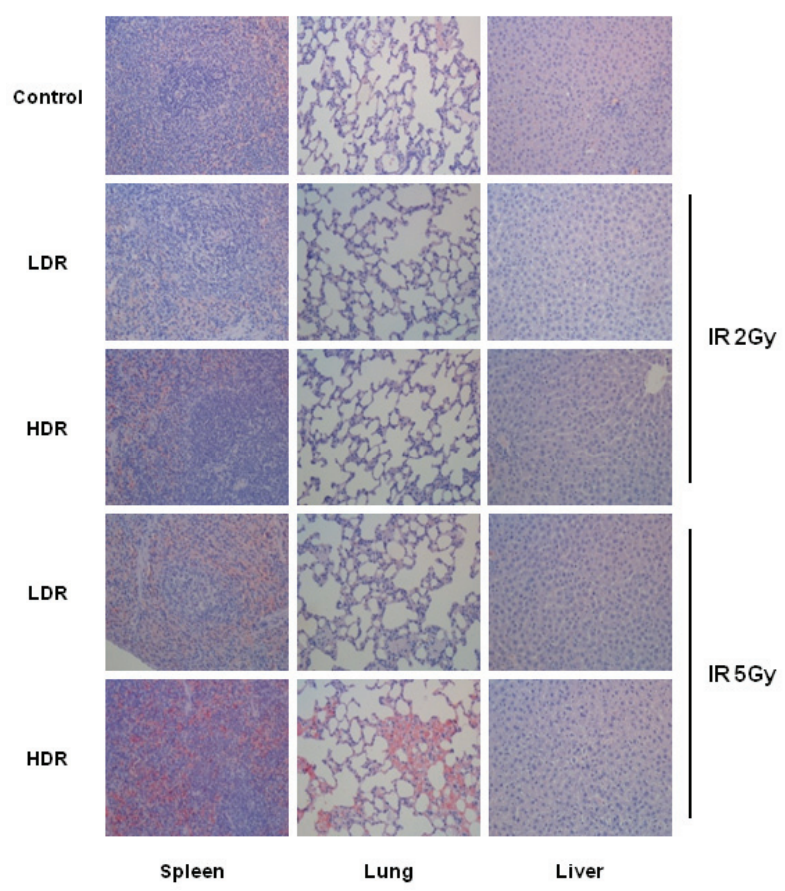

Fig. 2. Hematoxylin and eosin (H\&E)-stained sections of spleen, lung, and liver of irradiated rats. The figure shows representative microscopic images of rat spleen, lung, and liver after 2 Gy and 5 Gy administered as chronic LDR or acute HDR.
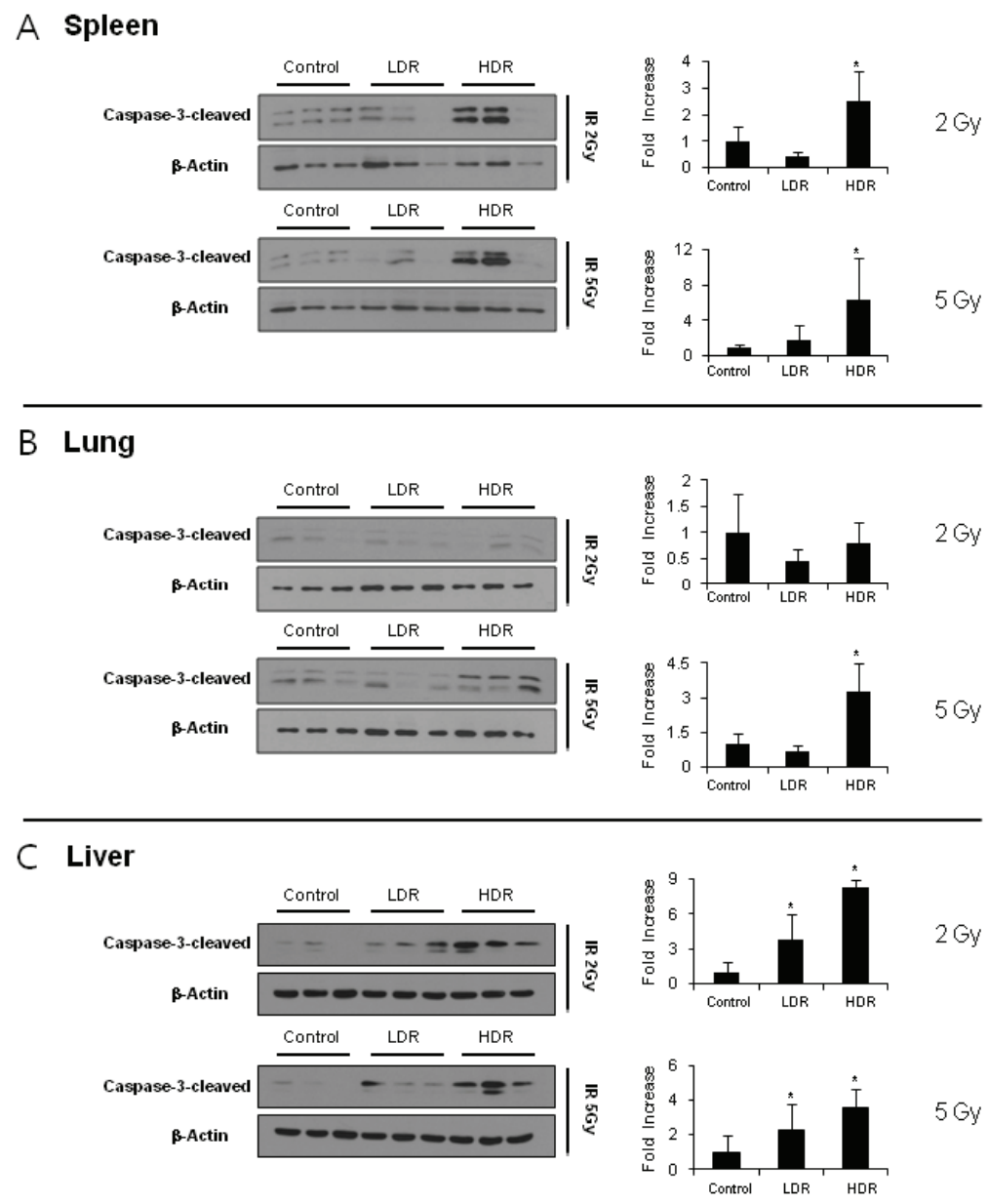

Fig. 3. Western blot analysis of caspase-3 cleavage in tissue from spleen (A), lung (B), and liver (C). After exposure of rats to chronic LDR or acute HDR of $2 \mathrm{~Gy}$ and $5 \mathrm{~Gy}$, lysates of spleen, lung, and liver tissues were analyzed by western blotting using antibody to the cleaved form of caspase-3. Each group included three rats. The relative protein band intensity was calculated from densitometric scans of the immunoblots with control values set at 1 . Equal protein loading was confirmed by $\beta$-actin expression and cleaved caspase- 3 expression was normalized to $\beta$-actin expression. ( $n=3$, data are presented as means \pm s.d.). 


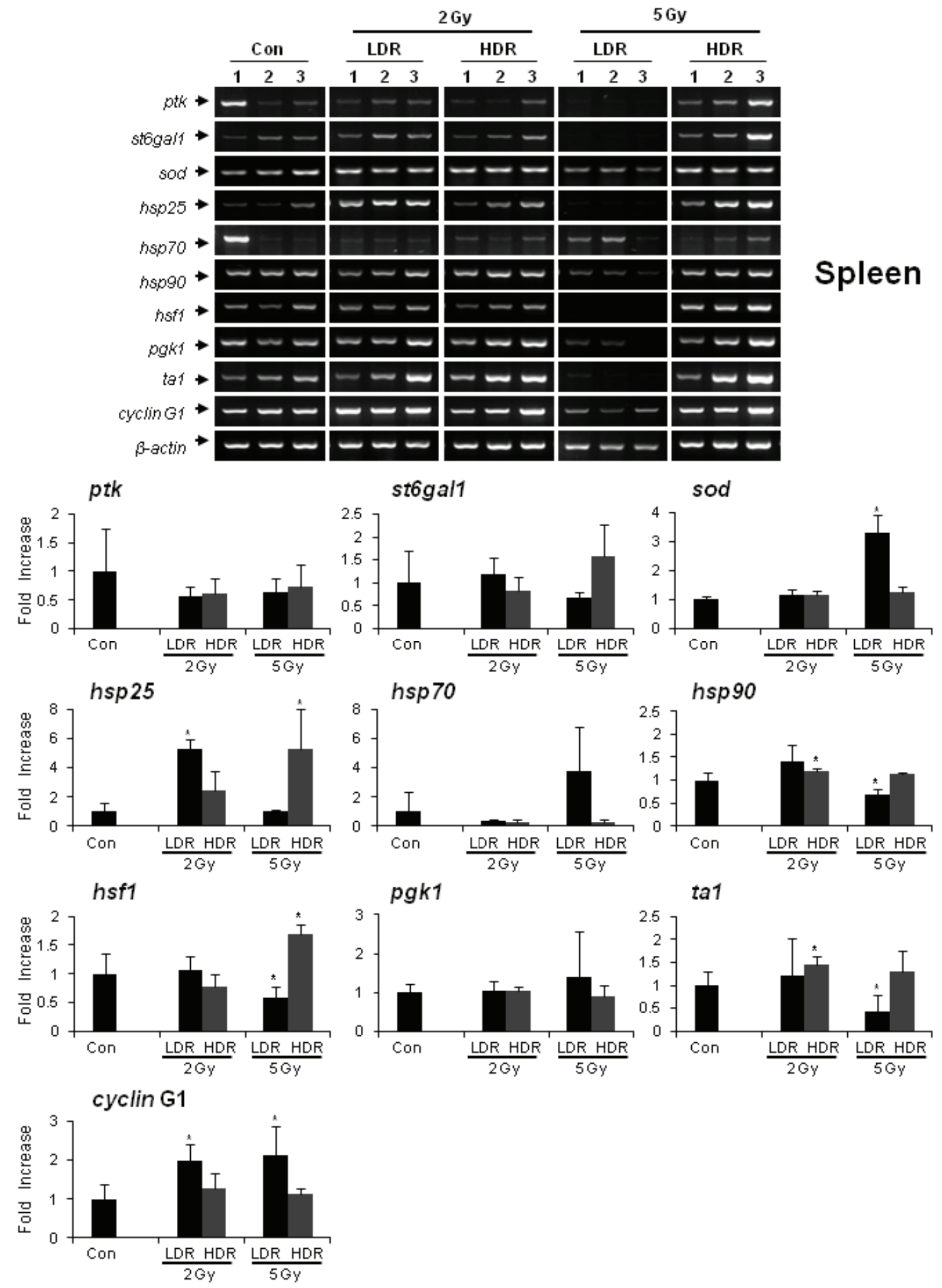

Fig. 4. RT-PCR analysis of mRNA expression of radiation-responsive genes in spleen tissues of rats after chronic LDR or acute HDR of $2 \mathrm{~Gy}$ and $5 \mathrm{~Gy}$. The relative mRNA band intensity was calculated from densitometric scans of the blots with control values set at 1. mRNA expression was normalized to that of $\beta$-actin $\left(\mathrm{n}=3\right.$, data are presented as means \pm s.d., $\left.{ }^{*} \mathrm{p}<0.05\right)$.

expression of $h s p 90$ and tal whereas HDR increased the expression of $h s f 1$ and $h s p 25$, compared with untreated control spleens. Therefore, cyclin G1 was the only gene that was differentially affected by LDR irrespective of dose and may be a candidate LDR-responsive gene in the spleen. The number of genes 


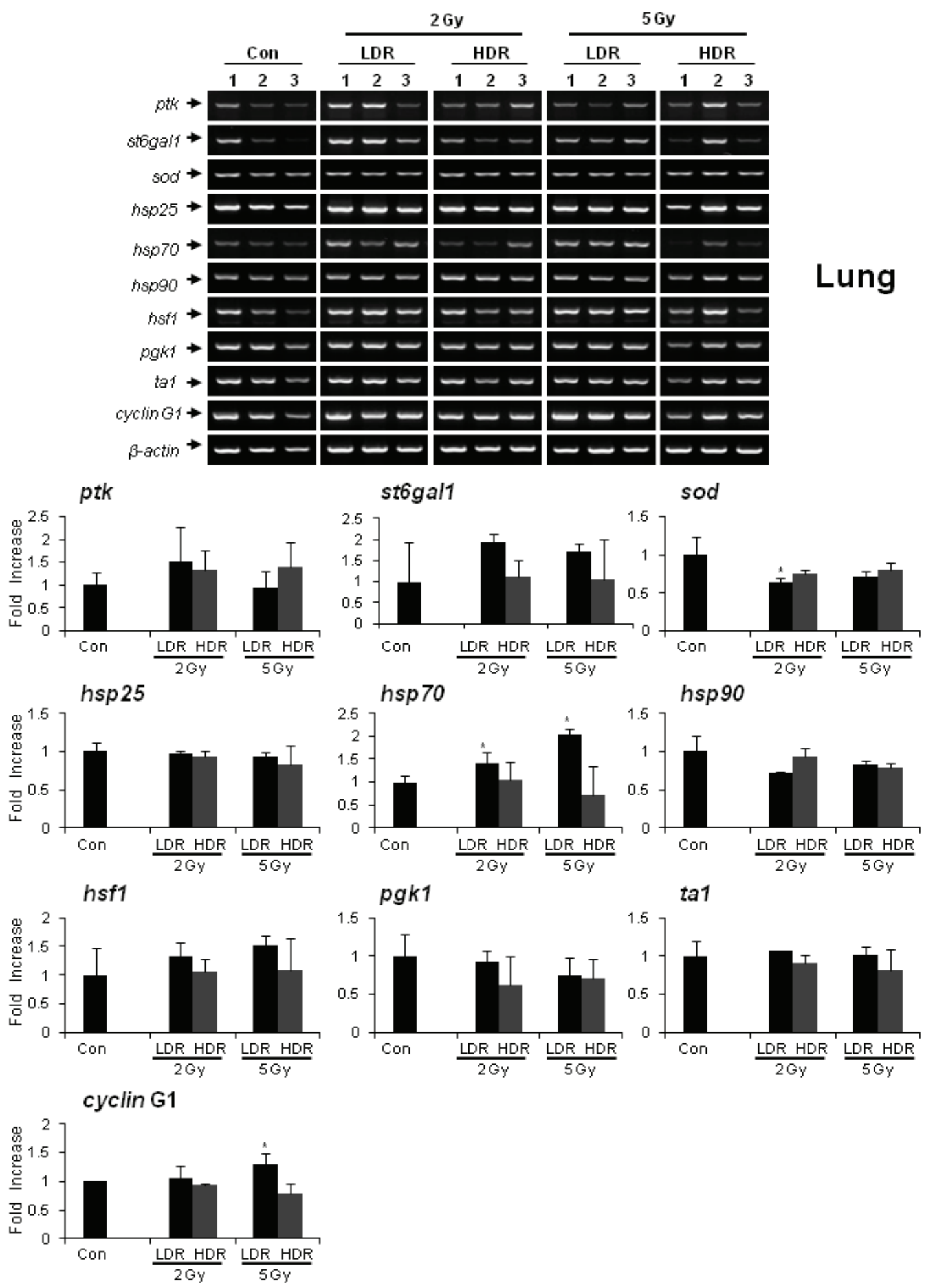

Fig. 5. RT-PCR analysis of mRNA expression of radiation-responsive genes in lung tissues of rats after chronic LDR or acute HDR of 2 Gy and 5 Gy. The relative mRNA band intensity was calculated from densitometric scans of the blots with control values set at 1 . mRNA expression was normalized to that of $\beta$-actin $\left(\mathrm{n}=3\right.$, data are presented as means \pm s.d., $\left.{ }^{*} \mathrm{p}<0.05\right)$.

whose expression was affected by LDR was greater than the number affected by HDR (Fig. 4).

3.4 Differential gene expression patterns in lung tissue according to LDR or HDR exposure
Lung tissue showed only marginal differences between HDR and LDR exposure with respect to histopathology and cleavage of caspase 3. No significant induction of cleaved caspase 3 was detected after HDR exposure at a dose of 2 Gy, but 5 Gy HDR 


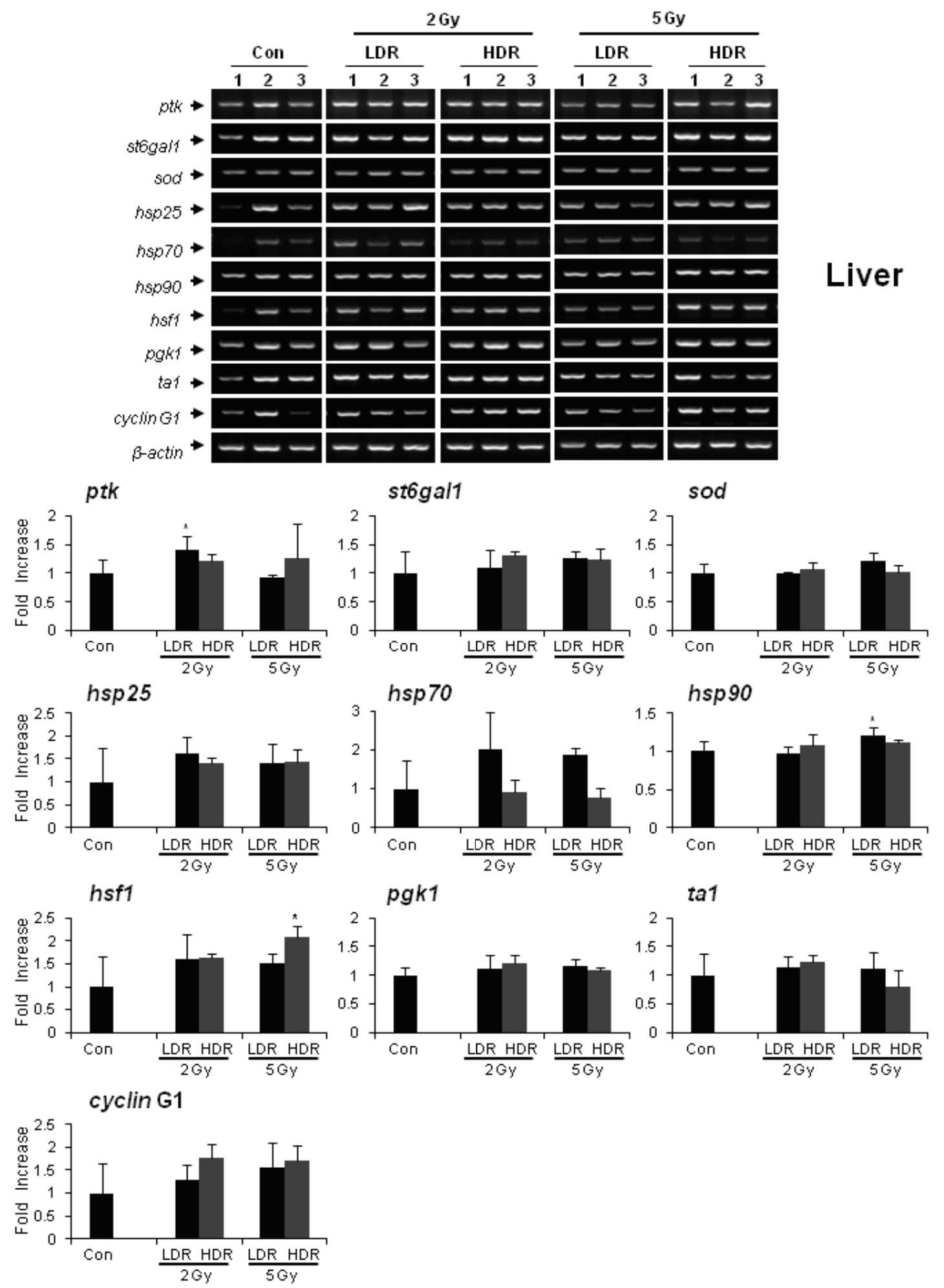

Fig. 6. RT-PCR analysis of mRNA expression level of radiation-responsive genes in liver tissues of rats after chronic LDR or acute HDR of $2 \mathrm{~Gy}$ and $5 \mathrm{~Gy}$. The relative mRNA band intensity was calculated from densitometric scans of the blots with control values set at 1. mRNA expression was normalized that of to $\beta$-actin $\left(\mathrm{n}=3\right.$, data are presented as means \pm s.d., $\left.{ }^{*} \mathrm{p}<0.05\right)$.

exposure significantly induced the cleavage of caspase 3. However, LDR exposure did not induce the cleavage of caspase 3 , even at the higher dose of 5 Gy. Analysis of gene expression patterns in the lung showed that 2 Gy LDR exposure decreased ex- pression of sod and increased expression of $h s p 70$ whereas 2 Gy HDR did not significantly alter gene expression patterns. In animals treated with a dose of 5 Gy, hsp70 and cyclin Gl were significantly overexpressed following LDR exposure and no gene was 
differently expressed upon HDR exposure. Similar to spleen tissue, LDR exposure resulted in a greater change in gene expression patterns than HDR exposure (Fig. 5).

\subsection{Differential gene expression patterns in liver tis- sue according to LDR or HDR exposure}

Liver tissue showed no differences between HDR and LDR exposure with respect to histopathology and both HDR and LDR exposure induced cleavage of caspase 3. At a dose of 2 Gy, neither LDR nor HDR exposure significantly altered gene expression patterns. LDR exposure at 5 Gy induced increased expression of $h s p 90$ and HDR exposure at 5 Gy increased expression of $h s f l$ (Fig. 6). Therefore, although LDR and HDR had a different effect on gene expression in the liver, the sensitivity of gene expression changes appeared to be similar between LDR and HDR exposure.

\section{DISCUSSION}

HDR radiation exposure can impair DNA repair and induce cancer or genetic disorders; however, less is known about the effect of LDR radiation. In this study, we compared histopathology, caspase 3 activation, and expression patterns of genes that were previously identified as being responsive to irradiation [19-21, 24] in spleen, lung, and liver tissues after acute (HDR) or chronic (LDR) exposure to ionizing radiation.

Spleen tissue showed the most dramatic differences between acute and chronic exposure. Chronic exposure produced little pathological change and minimal cell apoptosis in the spleen of irradiated rats compared with un-irradiated controls. In contrast, acute exposure induced serious pathologic changes and extensive apoptosis. This might be because spleen tissue contains many types of immune cells and LDR exposure may induce immunological activators that inhibit the radiation damage and apoptosis. This is supported by reports that immune cells show an adaptive response to low-dose radiation [25-28].

In lung tissue, acute exposure did not induce any significant increase in apoptosis at 2 Gy but did at 5 Gy, indicating a reduced sensitivity of lung tissue to IR compared with spleen tissue. Differences in pathologic changes and caspase 3 activation between chronic and acute exposure were apparent upon exposure to an IR dose of $5 \mathrm{~Gy}$, although neither acute nor chronic exposure at a dose of 2 Gy induced any serious damage or apoptosis in lung tissue and there was no appreciable difference between LDR and HDR at the low dose. Therefore, lung showed LDR effects at a higher dose than spleen tissue.

In the liver tissue there were no apparent differences between acute and chronic exposure in terms of histologic changes and apoptosis, and caspase 3 activation was induced by both acute and chronic exposure regardless of dose. These results suggest that the liver is a more sensitive organ than the lung. The lack of LDR effects in liver tissue suggests that, unlike the spleen, liver may not possess sufficient immune cell populations or the necessary factors to inhibit the induction of radiation damage and apoptosis by LDR exposure.

The present results support the hypothesis that the spleen may be a radiation-responsive organ that is affected by dose rate, with LDR responses predominating. In lung tissue, LDR effects were induced only by relatively high doses of IR whereas liver tissue showed no differences between LDR and HDR at high and low doses. One of the key cellular responses to radiation exposure is the induction of a pathway that leads to apoptosis. Interestingly, the relative amounts of apoptosis vary between different tissues, and genetic modifiers of radiation-induced apoptosis act differently in different tissues [29, 30], suggesting cell-type specificity for apoptosis. Moreover, some reports have described that dose rate is important for radiation damage responses such as apoptosis. The present study comparing radiation damage according to dose rate in different organs demonstrates for the first time that the spleen is a sensitive organ that is responsive to LDR exposure, whereas the liver is not affected by dose rate if the total dose is the same. In the lung, LDR effects were only seen at a relatively high dose.

We previously analyzed genes that are overexpressed in human peripheral blood lymphocytes [19] and examined their expression in brain, heart, spleen, intestine, and lung, organs that show different radiosensitivities [24], after low dose acute radiation exposure (HDR IR with $0.2 \mathrm{~Gy}$ ). We also examined the correlation between gene expression patterns and organ response by the induction of apoptosis and attempted to identify genes that might be responsible for any difference in radiation response. In an extension of these studies, in the current study we compared the expression patterns of radiation response genes according to LDR or HDR exposure. We found that expression of cyclin $G 1$ was upregulated in the spleen in response to LDR at both 2 Gy and 
5 Gy IR, whereas its expression was not induced by HDR exposure. Therefore, this gene may show a LDR-specific response in the spleen. Lung tissue also responded to 5 Gy LDR by up-regulation of cyclin G1 expression, but did not respond to LDR at the lower dose of 2 Gy. In liver tissue this gene was not responsive to LDR exposure, thus cyclin G1 expression correlated with pathologic and apoptosis findings. Cyclin G1 is a transcriptional target of p53 and is induced by DNA damage in a p53 dependent manner. Analysis of cyclin G1 disrupted mice demonstrated that cyclin G1 is involved in many of the functions regulated by p53 such as apoptosis, growth control and check point regulation in response to DNA damage. The results suggest that the main role of cyclin G1 is to mediate or regulate the function of p53 [31].

We failed to detect altered expression of the majority of selected genes following HDR exposure, even though these genes were identified as radiation-responsive genes in our previous studies [19-21, 24]. It is possible that doses of 2 Gy or 5 Gy HDR are too high to detect these genes or that earlier time points of examination are required since we previously identified these genes using low-dose IR (0.2 Gy and 1 Gy). Furthermore, some of the genes were significantly down-regulated, which may be next phenomena after activation.

In conclusion, LDR effects differed among lung, liver, and spleen tissues. The spleen showed the most differential response to HDR and LDR, and may undergo LDR-specific responses that reduce the radiation damage. Upregulated expression of cyclin $G 1$ may be involved in these LDR effects.

\section{Acknowledgements:}

This work was supported by a grant of the Nuclear R\&D Program (NRF2011-0031696 and NRF2013M 2A2A704043384) of the National Research Foundation of Korea (NRF), funded by the Korean government (Ministry of Science, ICT \& Future Planning).

\section{Declaration Of Interest}

The authors declare no competing financial interests.

\section{REFERENCES}

1. Luckey TD. Physiological benefits from low levels of ionizing radiation. Health Phys. 1982; 43(6):771-789.

2. Yonezawa M, Takeda A, Misonoh J. Acquired radioresistance after low dose $\mathrm{X}$-irradiation in mice. J Radiat Res. 1990;31(3):256-262.
3. Yonezawa M, Misonoh J, Hosokawa Y. Two types of $\mathrm{X}$-ray-induced radioresistance in mice: presence of 4 dose ranges with distinct biological effects. Mutat Res. 1996;358(2):237-243.

4. Ducoff HS. Form of the increased longevity of Tribolium after X-irradiation. Exp Gerontol. 1975;10(3-4):189-193.

5. Mine M, Okumura Y, Ichimaru M, Nakamura T, Kondo S. Apparently beneficial effect of low to intermediate doses of A-bomb radiation on human lifespan. Int J Radiat Biol. 1990;58(6): 1035-1043.

6. James SJ, Makinodan T. T cell potentiation in normal and autoimmune-prone mice after extended exposure to low doses of ionizing radiation and/or caloric restriction. Int $\mathrm{J}$ Radiat Biol Relat Stud Phys Chem Med. 1988;53(1): 137-152.

7. James SJ, Enger SM, Peterson WJ, Makinodan $\mathrm{T}$. Immune potentiation after fractionated exposure to very low doses of ionizing radiation and/or caloric restriction in autoimmune-prone and normal $\mathrm{C} 57 \mathrm{Bl} / 6$ mice. Clin Immunol Immunopathol. 1990;55(3):427-437.

8. Matsubara $\mathrm{J}$, Turcanu $\mathrm{V}$, Poindron $\mathrm{P}$, Ina $\mathrm{Y}$. Immune effects of low-dose radiation: short-term induction of thymocyte apoptosis and long-term augmentation of T-cell-dependent immune responses. Radiat Res. 2000;153(3):332-338.

9. Kojima S, Ishida $\mathrm{H}$, Takahashi M, Yamaoka K. Elevation of glutathione induced by low-dose gamma rays and its involvement in increased natural killer activity. Radiat Res. 2002;157(3): 275-280.

10. Lee YJ, Ducoff HS. Radiation factors and their influence on induction of oxygen resistance. Radiat Res. 1989;117(1):158-162.

11. Miyachi Y, Yamada T. Low-dose X-ray-induced depression of sexual behavior in mice. Behav Brain Res. 1994;65(1):113-115.

12. Shen RN, Lu L, Kaiser HE, Broxmeyer HE. Murine AIDS cured by low dosage total body irradiation. Adv Exp Med Biol. 1997;407: 451-458.

13. Hashimoto S, Shirato H, Hosokawa M, Nishioka T, Kuramitsu Y, Matushita K, Kobayashi M, Miyasaka K. The suppression of metastases and the change in host immune response after low-dose total-body irradiation in tumor-bearing rats. Radiat Res. 1999;151(6):717-724.

14. Takahashi M, Kojima S, Yamaoka K, Niki E. Prevention of type I diabetes by low-dose gamma 
irradiation in NOD mice. Radiat Res. 2000; 154(6):680-685.

15. Cardis E, Vrijheid M, Blettner M, Gilbert E, Hakama M, Hill C, Howe G, Kaldor J, Muirhead CR, Schubauer-Berigan M, Yoshimura T, Bermann F, Cowper G, Fix J, Hacker C, Heinmiller B, Marshall M, Thierry-Chef I, Utterback D, Ahn YO, Amoros E, Ashmore P, Auvinen A, Bae JM, Bernar J, Biau A, Combalot E, Deboodt P, Diez Sacristan A, Eklöf M, Engels $\mathrm{H}$, Engholm G, Gulis G, Habib RR, Holan K, Hyvonen H, Kerekes A, Kurtinaitis J, Malker H, Martuzzi M, Mastauskas A, Monnet A, Moser M, Pearce MS, Richardson DB, RodriguezArtalejo F, Rogel A, Tardy H, Telle-Lamberton M, Turai I, Usel M, Veress K. The 15-Country Collaborative Study of Cancer Risk among Radiation Workers in the Nuclear Industry: estimates of radiation-related cancer risks. Radiat Res. 2007;167(4):396-416.

16. Kadhim MA, Lorimore SA, Townsend KM, Goodhead DT, Buckle VJ, Wright EG. Radiation-induced genomic instability: delayed cytogenetic aberrations and apoptosis in primary human bone marrow cells. Int $\mathrm{J}$ Radiat Biol. 1995;67(3):287-293.

17. Morgan WF, Day JP, Kaplan MI, McGhee EM, Limoli CL. Genomic instability induced by ionizing radiation. Radiat. Res. 1996;146(3):247-258.

18. Suzuki K, Ojima M, Kodama S, Watanabe M. Radiation-induced DNA damage and delayed induced genomic instability. Oncogene. 2003; 22(45):6988-6993.

19. Kang CM, Park KP, Song JE, Jeoung DI, Cho CK, Kim TH, Bae S, Lee SJ, Lee YS. Possible biomarkers for ionizing radiation exposure in human peripheral blood lymphocytes. Radiat Res. 2003;159(3):312-319.

20. Lim YB, Pyun BJ, Lee HJ, Jeon SR, Jin YB, Lee YS. Proteomic identification of radiation response markers in mouse intestine and brain. Proteomics. 2011;11(7):1254-1263.

21. Lee HJ, Lee M, Kang CM, Jeoung D, Bae S, Cho CK, Lee YS. Identification of possible candidate biomarkers for local or whole body radiation exposure in $\mathrm{C} 57 \mathrm{BL} / 6$ mice. Int $\mathrm{J}$ Radiat Oncol Biol Phys. 2007;69(4):1272-1281.

22. Ruka W, Taghian A, Gioioso D, Fletcher JA, Preffer F, Suit HD. Comparison between the in vitro intrinsic radiation sensitivity of human soft tissue sarcoma and breast cancer cell lines. J Surg Oncol. 1996;61(4):290-294.
23. Tapio S. Ionizing radiation effects on cells, organelles and tissues on proteome level. Adv Exp Med Biol. 2013;990:37-48.

24. Lee WJ, Majumder ZR, Jeoung DI, Lee HJ, Kim $\mathrm{SH}$, Bae S, Lee YS. Organ-specific gene expressions in C57BL/6 mice after exposure to low-dose radiation. Radiat Res. 2006;165(5): 562-569.

25. Cheng GH, Wu N, Jiang DF, Zhao HG, Zhang Q, Wang JF, Gong SL. Increased levels of p53 and PARP-1 in EL-4 cells probably related with the immune adaptive response induced by low dose ionizing radiation in vitro. Biomed Environ Sci. 2010;23(6):487-495.

26. Ina $\mathrm{Y}$, Tanooka $\mathrm{H}$, Yamada $\mathrm{T}$, Sakai $\mathrm{K}$. Suppression of thymic lymphoma induction by life-long low-dose-rate irradiation accompanied by immune activation in C57BL/6 mice. Radiat Res. 2005;163(2):153-158.

27. Yu HS, Song AQ, Lu YD, Qiu WS, Shen FZ. Effects of low-dose radiation on tumor growth, erythrocyte immune function and SOD activity in tumor-bearing mice. Chin Med J (Engl). 2004; 117(7):1036-1039.

28. Gong SL, Liu SC, Liu JX, Zhang YC, Liu SZ. Adaptive response of thymocyte apoptosis and cell cycle progression induced by low dose X-ray irradiation in mice. Biomed Environ Sci. 2000;13(3):180-188.

29. Nomura $T$, Kinuta $M$, Hongyo $T$, Nakajima $H$, Hatanaka T. Programmed cell death in whole body and organ systems by low dose radiation. J. Radiat Res. 1992;33 Suppl:109-123.

30. Mori N, van Wezel T, van der Valk M, Yamate J, Sakuma S, Okumoto M, Demant P. Genetics of susceptibility to radiation-induced apoptosis in colon: two loci on chromosomes 9 and 16 . Mamm. Genome. 1998;9(5):377-380.

31. Kimura SH, Nojima H. Cyclin G1 associates with MDM2 and regulates accumulation and degradation of p53 protein. Genes Cells. 2002;7(8): 869-880. 